\title{
Parallel Regulation of Past, Present, and Future Actions During Sequencing
}

\author{
Lawrence P. Behmer Jr. ${ }^{1}$, Kelly J. Jantzen ${ }^{2}$, Sarah Martinez ${ }^{2}$, Rachel Walls ${ }^{2}$, Elisabeth Amir-Brownstein ${ }^{2}$, \\ Andrew Jaye ${ }^{2}$, Mckaila Leytze ${ }^{2}$, Kathleen Lucier ${ }^{2}$, \& Matthew J. C. Crump ${ }^{1,3}$ \\ ${ }^{1}$ Department of Psychology, Brooklyn College of CUNY \\ 2 Department of Psychology, Western Washington University \\ ${ }^{3}$ Department of Psychology, Graduate Center of CUNY
}

\begin{abstract}
Past, present, and future actions must be regulated online to produce sequences of actions, but the regulation process is not well understood because of measurement limitations. We provide the first direct tests of the parallel action regulation hypothesis during sequencing in humans. We used transcranial magnetic stimulation to probe the level of excitation for flexion of the right index finger during typing. Motor evoked potentials (MEPs) were recorded at the onset of typing 5-letter words and nonwords. A single letter typed by the right index finger varied across letter positions 1 to 5. MEP amplitude was largest for the upcoming action in the second position and decreased monotonically across future serial positions, suggesting a serial inhibition process regulates all future actions in parallel during sequencing. This is the most direct human evidence to date corroborating models of sequence production that assume parallel regulation of actions.
\end{abstract}

Keywords: action sequencing, serial order, motor evoked potentials, typing, transcranial magnetic stimulation

Word count: Just enough, see next page for citation info, onlineFirst @ JEP : HPP

People sequence actions to accomplish a variety of performance goals. Actions must be regulated so that completed actions are not repeated, present actions are executed, and future actions are correctly ordered (see Behmer \& Crump, 2017). Previous research using typists (Gentner, Grudin, \& Conway, 1980) suggests an online parallel process regulates action states for all actions in a sequence. However, direct measures are needed to test how the activation states of past, present, and future actions are regulated during sequencing. We used transcranial magnetic stimulation (TMS) and motor evoked potentials (MEPs) to measure parallel activation of any action in a sequence, during a typing task, and provide novel evidence of parallel action regulation in humans.

Competitive queuing models (see Figure 1) can simulate typewriting (Rumelhart \& Norman, 1982) and propose that all actions are activated in parallel, with each action inhibiting all successive actions in the sequence (Estes, 1972; Grossberg, 1987). In empirical studies, direct evidence of parallel action regulation was observed in monkeys by measuring neural activity during line-segment production in a shape drawing task (Averbeck, Chafee, Crowe, \& Georgopoulos, 2002). Neurons associated with each line segment showed parallel activity during planning, and firing rates increased in series as segments were produced. In humans, our previous work showed indirect evidence using reactions times (RTs): Skilled typists show parallel activation of all keystrokes for letters in a word but not random strings (Crump \& Logan, 2010), and when typists were occasionally cued to type letters shifted one to three letters ahead or behind the next expected letter, keystroke times increased as a function of serial position, consistent with serial inhibition (Behmer \& Crump, 2017).

Ideally, testing predictions about parallel action regulation in humans requires direct concurrent measures of action activation states for all actions during sequencing. We created a "thermometer" to "take the temperature" of activation states of current and future actions during typing. We applied single-pulse TMS over the motor cortex to measure motor readiness from MEPs at the right index finger during typing (Fadiga, Fogassi, Pavesi, \& Rizzolatti, 1995). Typists copied words and random strings constructed so the right index finger was not used (all left-hand letters) or used once across the five serial positions. TMS was triggered at the onset of the first keystroke. Thus, MEPs were measured when the right index finger was a completed response (position 1), a future response (positions 2-5), or a nonresponse (see Figure 2).

We asked the following: (1) Do MEPs show decreasing amplitudes across serial position consistent with parallel activation and serial inhibition across all actions? (2) Are MEPs for the completed action in the first position smaller than the second position, indicating rapid deactivation? (3) Is the range of partially activated future actions wider for words than 
random strings? Typists produce words faster than random strings (Gentner, Larochelle, \& Grudin, 1988), potentially because words cause parallel activation of letters, and random strings do not (Crump \& Logan, 2010), so keystrokes may only be regulated in parallel for words.

\section{Method}

\section{Participants}

Fourteen participants ( 7 females) from Western Washington University participated in the experiment $(M=21.8$, $\mathrm{SD}=2.8$ years). The Western Washington University institutional review board approved the study. All participants were strongly right-handed (Oldfield, 1971).

\section{Materials}

Words or random strings were constructed so the right index finger was used once across the five serial positions (RiLLLL, LRiLLL, LLRiLL, LLLRiL, LLLLRi) or not at all (LLLLL). Five-letter English words (familiarity range: 100700) were taken from the MRC Psycholinguistic Database (Wilson, 1987). Random strings were generated by sampling letters randomly without replacement using $\mathrm{R}$. We selected words and random strings where the letters $\mathrm{H}, \mathrm{M}, \mathrm{N}$, or $\mathrm{U}$ (all typed by the right index finger) occurred in serial positions $1-4$, and the letters $\mathrm{H}, \mathrm{M}$, and $\mathrm{N}$, occurred at serial position 5 . Across strings, each letter occurred in each position. Within a string, the letter assigned to the right index finger appeared

This preprint was made in $\mathrm{R}$ using papaja, and is onlineFirst at the Journal of Experimental Psychology : Human Perception and Performance. The citation is, Behmer, L. P., Jr., Jantzen, K. J., Martinez, S., Walls, R., Amir-Brownstein, E., Jaye, A., Leytze, M., Lucier, K., \& Crump, M. J. C. (2018, July 19). Parallel Regulation of Past, Present, and Future Actions During Sequencing. Journal of Experimental : Psychology Human Perception and Performance. Advance online publication. http://dx.doi.org/10.1037/xhp0000507

Lawrence P. Behmer Jr., Department of Psychology, Brooklyn College of the City University of New York; Kelly J. Jantzen, Sarah Martinez, Rachel Walls, Elisabeth Amir-Brownstein, Andrew Jaye, Mckaila Leytze, and Kathleen Lucier, Department of Psychology, Western Washington University; Matthew J. C. Crump, Department of Psychology, Brooklyn College of the City University of New York, and Graduate Center of the City University of New York.

This work was supported by a grant from the National Science Foundation (principal investigator, Matthew J. C. Crump, 1353360). This observation is a follow-up with a new direct measure of response set activation that provides converging evidence for the findings from our full report (Behmer \& Crump, 2017).

Correspondence concerning this article should be addressed to Lawrence P. Behmer Jr., Department of Psychology, Brooklyn College of the City Univer- sity of New York, 2900 Bedford A venue, Brooklyn, NY 11210. E-mail: LBehmer@brooklyn.cuny.edu

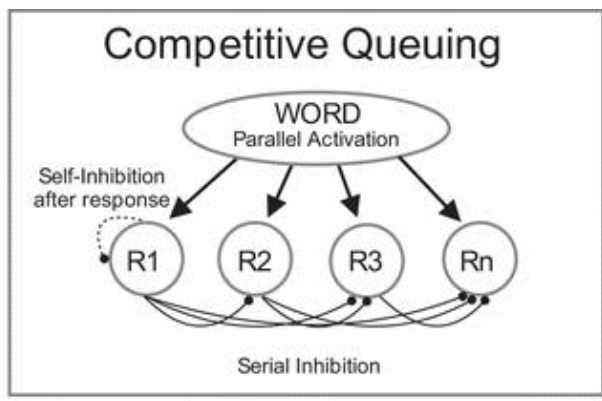

Predicted Response Activation

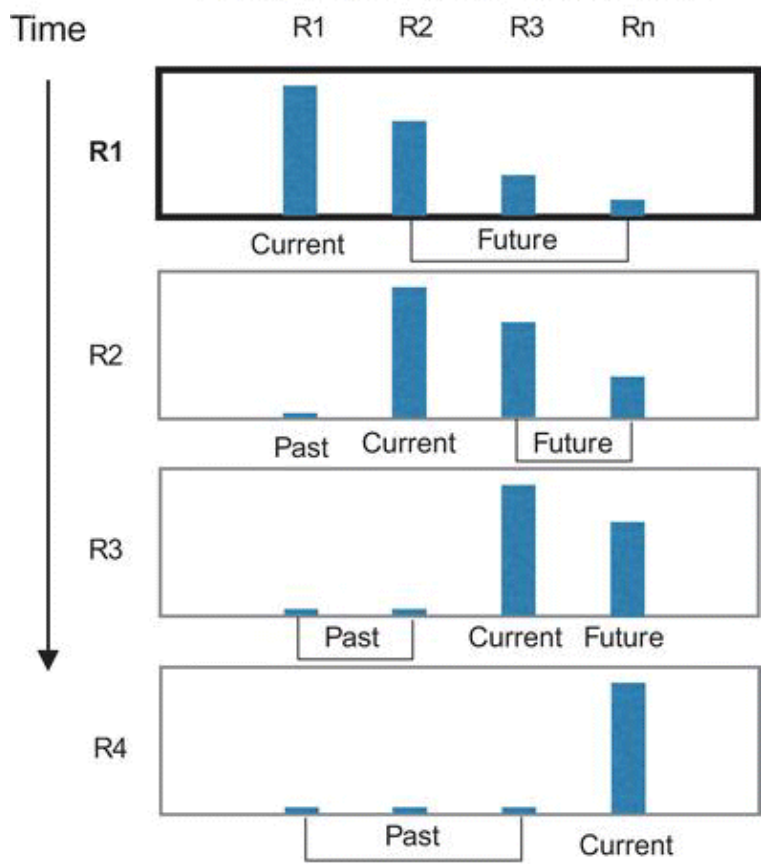

Figure 1. Competitive queuing models assume that all responses are first activated in parallel, and then each action inhibits all following actions. The predicted activation states for each action are shown across time as each current (R1 to $\mathrm{R} 4$ ) is completed. The critical prediction is that future activation states are partially activated in a monotonic, decreasing fashion across serial position.

once, and the right index finger was used once per string. Baseline strings contained all left-hand letters. We excluded strings with letters $\mathrm{Y}$ and $\mathrm{J}$, which are typed with the right index finger but did not occur in our word set across each of the five serial positions. We also excluded strings with lefthand letters T, G, B, and V because subjects inconsistently used their right index finger to type those letters during pilot testing.

\section{Design and Procedure}

The experiment was controlled in-house by LiveCode 8.1. We used a 2 (string type: word vs. random) by 6 (serial 
position: 1-5 and baseline) design. Each participant typed 72 word and 72 random strings. String type was blocked. For serial position conditions $1-4$, three words were randomly selected from each bin in which the letters, $\mathrm{H}, \mathrm{M}, \mathrm{N}$, and $\mathrm{U}$ occurred once, at each position. For words that ended with a right index finger response (position 5), four words were randomly selected from each bin in which the letters, H, M, and $\mathrm{N}$ occurred once, at each position. Serial position was randomized across trials.

Each trial began with a 2,000-ms fixation cross followed by a string appearing in a gray textbox above the fixation cross (18-point, black, Times New Roman font). Participants were instructed to type the string as quickly and accurately as possible. Their output appeared in a white textbox below fixation. Backspacing was disabled and participants were instructed to type the next character following an error. After five keypresses, a blank gray screen was presented for $2 \mathrm{~s}$, followed by the next trial. There was a short break between blocks.

\section{TMS}

TMS was applied to the contralateral motor cortex using a Magstim BiStim2 (Whitland, Wales) outfitted with a 70-mm figure 8 coil. A neuronavigation suite (Rogue Research, Montreal, Canada) tracked the position of the coil and participant's head in real time. We determined the target scalp location that produced a maximal MEP in the first dorsal interosseous muscle using an iterative search procedure. Each of 25 points on a $5-\mathrm{cm} \times 5-\mathrm{cm}$ search grid centered on the scalp location C3 of the International 10-20 system was targeted with a single pulse at $40 \%-50 \%$ of maximum simulator output. The coil was directed from posterior to anterior and rotated around the vertical axis at an angle of approximately 45 degrees relative to a line running anterior/posterior along the central midline. The location producing the largest MEP from the first dorsal interosseous (FDI) was used as the center for a second, finer search grid $(25$ points at $2.5 \times 2.5 \mathrm{~cm})$. The location on this second grid resulting in the largest MEP was used as the target for the remainder of the experiment. The three-dimensional location and orientation of the coil was recorded and maintained throughout the entire experiment.

The stimulator output was determined as $120 \%$ of active motor threshold (AMT). Participants gently squeezed a small object between the index finger and thumb of their right hand while six TMS pulses were delivered to the target location. Stimulator output was lowered from $50 \%$ of maximum until an MEP of at least $100 \mathrm{uV}$ was observed on no more than three of the six trials. The stimulator output for experimental trials was initially set at $120 \%$ of this value. If $120 \%$ of AMT produced noticeable movement of the index finger, the stimulation level was slowly decreased until movements were no longer evident. MEPs were recorded using an active electrode system (Biosemi, Amsterdam, Netherlands). Bipolar
$\mathrm{Ag}-\mathrm{AgCl}$ recording electrodes were placed on the right index finger with one electrode over the belly of the FDI and the other on the medial side of the finger, over the middle knuckle. Common Mode Sense and Driven Right Leg electrodes were placed on the anterior surface of the forearm. A continuous record was amplified and digitized at 2,048 $\mathrm{Hz}$ per channel. Event markers indicating the onsets of each trial and each keystroke were embedded in the digital record.

\section{Power Analysis}

Previous research suggests the standard deviation of MEP magnitude for a participant is $10 \%-15 \%$ of standardized amplitude (e.g., Darling, Wolf, \& Butler, 2006). A power analysis shows that assuming $15 \%$ variability, nine participants are required to detect a $20 \%$ change in MEP magnitude $90 \%$ of the time. A $20 \%$ change in MEP amplitude can be observed even when muscle activation is very small (Darling et al., 2006). If we assume worse data than expected and repeat the analysis with $20 \%$ variability, 13 participants are required to achieve the same power level for a $20 \%$ change. We sought to record from 15 participants and ended with usable data from 13 participants.

\section{Results}

The electromyography (EMG) record, measured as the difference between the bipolar electrode, was high pass filtered above $10 \mathrm{~Hz}$. The MEP on each trial was quantified within a 50-ms window beginning at the first keystroke onset. An MEP was not included in the analysis if there was a clear indication from the EMG for that trial that (a) the FDI was not engaged or engaged at the wrong time during trials requiring the right hand, or (b) the FDI was engaged on trials not requiring use of the right hand. MEP amplitude per trial was determined as the maximum peak-to-peak amplitude within the analysis window. MEP amplitudes were averaged by condition for each participant. MEP data were analyzed only for correctly spelled words ( $85 \%$ of normal and $78 \%$ of random string trials), and mean MEPs by condition are displayed in Figure 3. The behavioral results showed normal typing performance (see online supplemental materials).

A $2 \times 6$ (String Type $\times$ Serial Position) analysis of variance confirmed a main effect for string type, $\mathrm{F}(1,12)=5.66, \mathrm{p}$ $=.035, \eta \mathrm{p} 2=0.32$; serial position, $\mathrm{F}(5,60)=29.33, \mathrm{p}<.001$, $\eta \mathrm{p} 2=0.71$; and a String Type $\times$ Serial Position interaction, $\mathrm{F}(5,60)=5.85, \mathrm{p}=.004, \eta \mathrm{p} 2=0.33$. Multiple comparisons for the main effect and interactions were corrected at $\mathrm{p}<.005$ and $\mathrm{p}<.002$, respectively (see Table 1 ).

Our first question was whether MEP amplitudes decreased across serial position, consistent with parallel activation and serial inhibition across all actions. We compared position-by-position changes in MEP amplitude for future actions across positions 2-5 and between each future response 


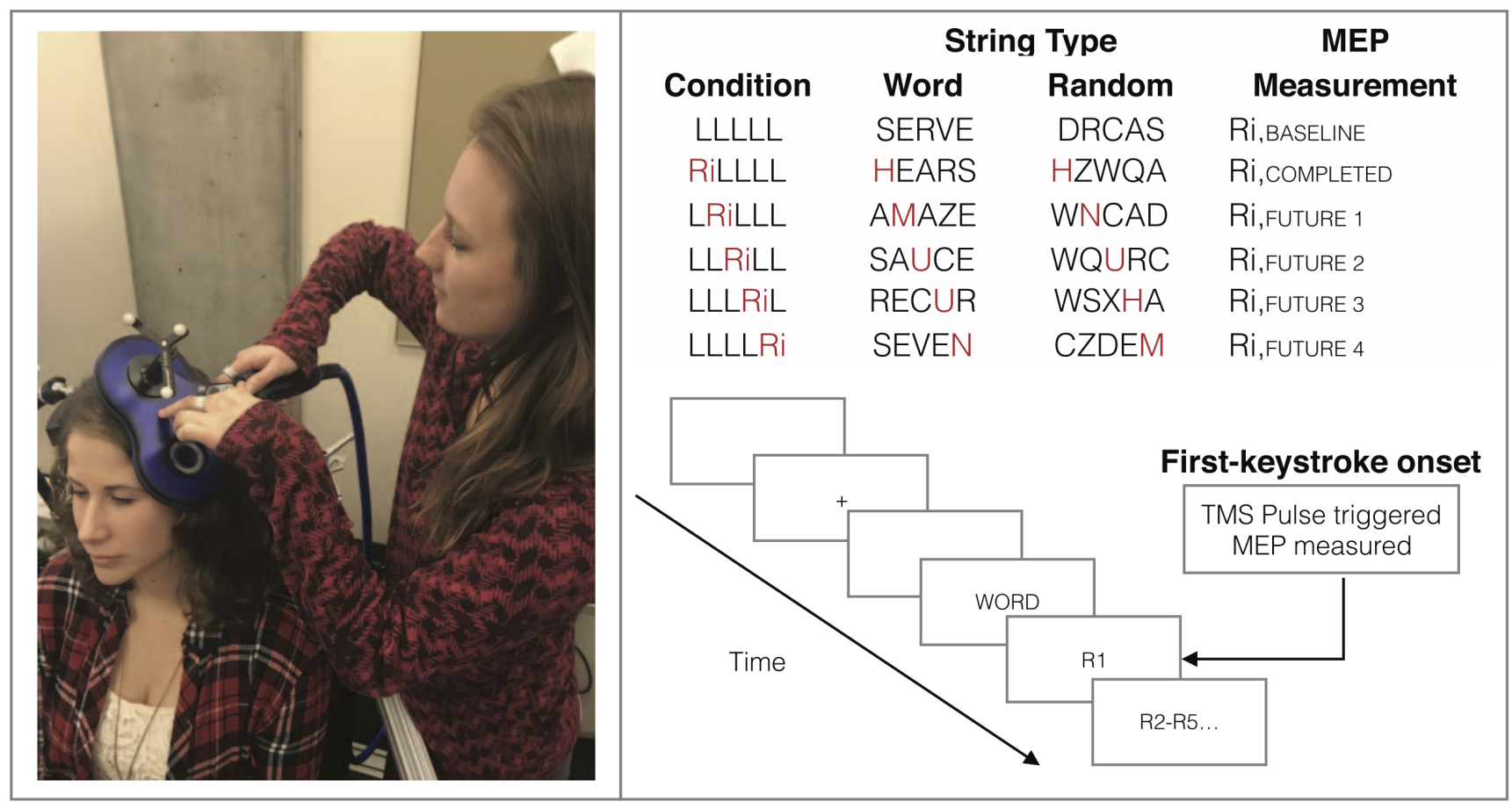

Figure 2. Subjects typed words or random strings on each trial. The transcranial magnetic stimulation (TMS) pulse was always triggered at the onset of the first keystroke, and the resulting motor evoked potential (MEP) was measured from the right index finger. Across trials, the right index finger was used once in the first to fifth serial position or was never used (baseline condition).

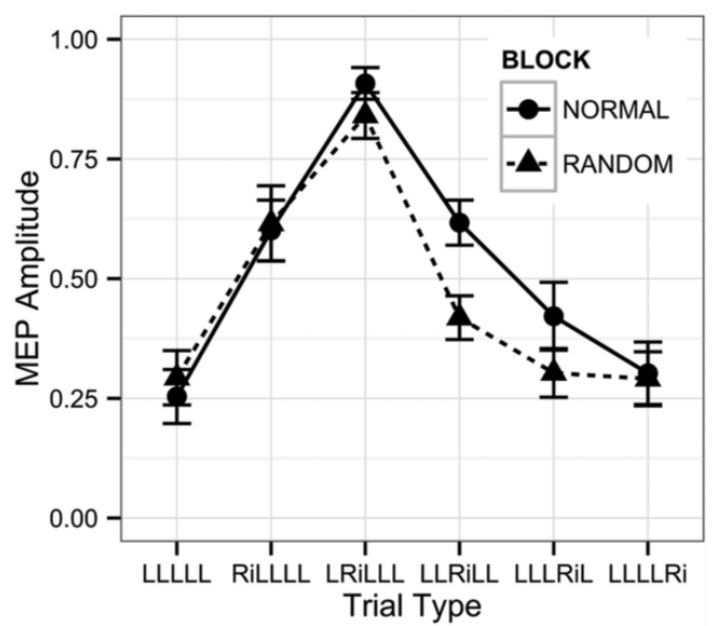

Figure 3. Mean motor evoked potential (MEP) amplitudes with standard error bars for normal and random strings across baseline (LLLLL) and serial positions 1 (RiLLLL), 2 (LRiLLL), 3 (LLRiLL), 4 (LLLRiL), and 5 (LLLLRi). L refers to lefthand letters, and Ri refers to letters completed by the right index finger. position with control trials that did not require a right index finger response (see Table 1 for all $t$ test comparisons). Positionby-position comparisons showed larger MEPs for the second (LRiLLL: $\mathrm{M}=0.87, \mathrm{SD}=0.15$ ) than third position (LLRiLL: $\mathrm{M}=0.52, \mathrm{SD}=0.19$ ), third than fourth position (LLLRiL: $\mathrm{M}$ $=0.36, \mathrm{SD}=0.23$ ), and fourth than fifth position (LLLLRi: $\mathrm{M}=0.30, \mathrm{SD}=0.22$ ). Comparisons against baseline (LLLLL: $\mathrm{M}=0.27, \mathrm{SD}=0.20$ ) showed larger MEPs when the right index finger was used in the first position (RiLLLL: $\mathrm{M}=$ $0.61, \mathrm{SD}=0.25$ ), second position, third position, and fourth position but not the fifth position. The pattern fits predictions of competitive queuing models (Estes, 1972; Rumelhart \& Norman, 1982), which predict that early responses should be more active compared to later responses in a sequence. The data are consistent with prior animal (Averbeck et al., 2002) and human studies (Behmer \& Crump, 2017; Crump \& Logan, 2010) showing parallel regulation of actions.

Our second question was whether MEPs would be smaller for the completed action in the first position than the next planned action in the second position, indicating evidence for rapid deactivation of a completed response, which is widely assumed by most sequence production models (for a recent review, see Hurlstone \& Hitch, 2015). Indeed, we found that MEPs were lower for completed responses in the first position compared to upcoming response in the second 
Table 1

$t$ Tests for Comparisons

\begin{tabular}{|c|c|c|c|c|}
\hline Comparison & $d f$ & $t$ value & $p$ value & Cohen's $d$ \\
\hline \multicolumn{5}{|l|}{ Main-effect comparisons } \\
\hline RiLLLL vs. LRiLLL & 12 & -3.00 & .011 & 1.38 \\
\hline LRiLLL vs, LLRiLL & 12 & 6.57 & $<.001$ & 2.48 \\
\hline LLRiLL vs. LLLRiL & 12 & 5.73 & $<.001$ & .88 \\
\hline LLLRiL vs. LLLLRi & 12 & 2.26 & .043 & .32 \\
\hline LLLLL vs. RiLLLL & 12 & -3.92 & .002 & 1.52 \\
\hline LLLLL vs. LRiLLL & 12 & -10.00 & $<.001$ & 3.57 \\
\hline LLLLL vs. LLRiLL & 12 & -9.68 & $<.001$ & 1.39 \\
\hline LLLLL vs. LLLRiL & 12 & -3.40 & .005 & .45 \\
\hline LLLLL vs, LLLLRi & 12 & -1.66 & .128 & .11 \\
\hline \multicolumn{5}{|l|}{ Interaction comparisons } \\
\hline Normal RiLLLL vs. Normal LRiLLL & 12 & -4.12 & .001 & 1.69 \\
\hline Normal LRiLLL vs. Normal LLRiLL & 12 & 5.40 & $<.001$ & 1.89 \\
\hline Normal LLRiLL vs. Normal LLLRiL & 12 & 4.63 & $<.001$ & .90 \\
\hline Normal LLLRiL vs. Normal LLLLRi & 12 & 3.20 & .008 & .48 \\
\hline Normal LLLLL vs. Normal RiLLLL & 12 & -4.36 & $<.001$ & 1.61 \\
\hline Normal LLLLL vs. Normal LRiLLL & 12 & -11.77 & $<.001$ & 3.93 \\
\hline Normal LLLLL vs. Normal LLRiLL & 12 & -10.41 & $<.001$ & 1.94 \\
\hline Normal LLLLL vs. Normal LLLRiL & 12 & -4.26 & .001 & .73 \\
\hline Normal LLLLL vs. Normal LLLLRi & 12 & -2.16 & .051 & .22 \\
\hline Random RiLLLL vs. Random LRiLLL & 12 & -2.02 & .066 & .96 \\
\hline Random LRiLLL vs. Random LLRiLL & 12 & 6.20 & $<.001$ & 2.50 \\
\hline Random LLRiLL vs. Random LLLRiL & 12 & 2.79 & .016 & .66 \\
\hline Random LLLRiL vs. Random LLLLRi & 12 & .33 & .746 & .06 \\
\hline Random LLLLL vs. Random RiLLLL & 12 & -3.23 & .007 & 1.30 \\
\hline Random LLLLL vs. Random LRiLLL & 12 & -8.08 & $<.001$ & 2.89 \\
\hline Random LLLLL vs. Random LLRiLL & 12 & -4.09 & .001 & .67 \\
\hline Random LLLLL vs. Random LLLRiL & 12 & -.36 & .722 & .05 \\
\hline Random LLLLL vs. Random LLLLRi & 12 & .12 & .902 & .01 \\
\hline Normal LLLLL vs. Random LLLLL & 12 & -1.25 & .235 & .19 \\
\hline Normal RiLLLL vs. Random RiLLLL & 12 & -.30 & .769 & .05 \\
\hline Normal LRiLLL vs. Random LRiLLL & 12 & 1.95 & .076 & .45 \\
\hline Normal LLRiLL vs. Random LLRiLL & 12 & 5.11 & $<.001$ & 1.19 \\
\hline Normal LLLRiL vs. Random LLLRiL & 12 & 2.10 & .057 & .53 \\
\hline Normal LLLLRi vs. Random LLLLRi & 12 & .35 & .733 & .05 \\
\hline
\end{tabular}

Note. Bold $p$ values for main effect $p<0.005$, and interaction at $p<0.002$.

Figure 4. t-tests for comparisons

position.

Our last question was whether the range of partially activated future actions would be wider for words than random strings. This question addresses whether word-level representations allow typists to activate all keystrokes in parallel. We analyzed MEPs for normal and random strings at positions 2-5 where the right index finger was planned as a future response. For normal strings, MEP amplitude was greater for the second $(\mathrm{M}=0.91, \mathrm{SD}=0.12)$ than third position $(\mathrm{M}=0.62, \mathrm{SD}=0.17)$, the third than fourth position $(\mathrm{M}=$ $0.42, \mathrm{SD}=0.26)$, and the fourth than fifth position $(\mathrm{M}=0.30$, $\mathrm{SD}=0.24)$. Additionally, MEPs were greater compared to baseline $(\mathrm{M}=0.25, \mathrm{SD}=0.50)$ for responses planned for the second, third, and fourth positions. Conversely, for random 
strings, MEP amplitude was only greater for the second (M $=0.84, \mathrm{SD}=0.17)$ compared to third position $(\mathrm{M}=0.42$, $\mathrm{SD}=0.16)$. Additionally, MEPs were greater than baseline $(\mathrm{M}=0.29, \mathrm{SD}=0.20)$ for responses planned for the second and third positions. Finally, we observed a difference in MEP amplitude between normal and random strings only when the right index finger was planned for the third position. We found a wider range of MEP amplitudes across serial positions for words than random strings, consistent with the idea that more keystrokes are regulated in parallel for words than random strings. Logan and Crump (2011) posited that skilled typewriting is controlled by two independently nested feedback loops, an outer loop that breaks down paragraphs and sentences into words and an inner loop that receives words and transforms them into letters and keystrokes. When typing random strings, the outer loop may be forced to plan responses serially, one or two letters at a time, and as a consequence, the range of parallel activation for random compared to normal strings may be less robust in comparison.

\section{Discussion}

We validated a novel TMS/MEP method showing the first direct evidence of parallel action regulation during sequence production in humans. The major results confirmed predictions of competitive queuing models. For planned actions, MEP amplitudes reflecting motor readiness decreased monotonically across serial positions $2-5$, consistent with a serial inhibition process. Completed actions in the first position showed smaller MEP amplitudes than the next planned response, consistent with a rapid deactivation process. In addition, the range of partial activations was wider for words than random strings, suggesting that the ability to regulate actions in parallel depends on experience. Importantly, these direct measures provide converging evidence consistent with recent behavioral findings (Behmer \& Crump, 2017).

Future work can use this method to understand the mechanisms of parallel action regulation and differentiate between models of sequence production. From the perspective of competitive queuing models, the time course of the transition between parallel activation and serial inhibition can be established by triggering TMS pulses at varying intervals after the onset of the word. If a wave of parallel activation occurs prior to serial inhibition, then all upcoming actions should be equally activated during the parallel wave and partially activated only after serial inhibition is applied. Serial recurrent network models (Elman, 1990) predict that actions should be activated as a function of their learned likelihood of occurrence given the preceding context of recent actions. Our method could test whether MEPs for particular future keystrokes vary as a function of their likelihood of occurrence based on the sequential structure of letter sequences in English or sequential structure of experimentally controlled strings. Oscillator-based timing models (Brown, Preece, \&
Hulme, 2000) assume that action states are controlled by timing signals that rise and fall in time when particular actions are required. Our method could be extended to rhythmic sequence production tasks to determine how parallel regulation processes govern temporally precise action control.

\section{References}

Averbeck, B. B., Chafee, M. V., Crowe, D. A., \& Georgopoulos, A. P. (2002). Parallel processing of serial movements in prefrontal cortex. Proceedings of the National Academy of Sciences of the United States of America, 99, 13172-13177. 10.1073/pnas.162485599

Behmer, L. P., Jr., \& Crump, M. J. C. (2017). The dynamic range of response set activation during action sequencing. Journal of Experimental Psychology: Human Perception and Performance, 43, 537-554. 10.1037/xhp0000335

Brown, G. D. A., Preece, T., \& Hulme, C. (2000). Oscillatorbased memory for serial order. Psychological Review, 107, 127-181. 10.1037/0033-295X.107.1.127

Crump, M. J. C., \& Logan, G. D. (2010). Hierarchical control and skilled typing: Evidence for word-level control over the execution of individual keystrokes. Journal of Experimental Psychology: Learning, Memory, and Cognition, 36, 1369-1380. 10.1037/a0020696

Darling, W. G., Wolf, S. L., \& Butler, A. J. (2006). Variability of motor potentials evoked by transcranial magnetic stimulation depends on muscle activation. Experimental Brain Research, 174, 376-385. 10.1007/s00221-006-0468-9

Elman, J. L. (1990). Finding structure in time. Cognitive Science, 14, 179-211. 10.1207/s15516709cog1402_1

Estes, W. K. (1972). An associative basis for coding and organization in memory. In A. W. Melton \& E. Martin (Eds.), Coding processes in human memory (pp. 161190). Washington, DC: V.H. Winston \& Sons.

Fadiga, L., Fogassi, L., Pavesi, G., \& Rizzolatti, G. (1995). Motor facilitation during action observation: A magnetic stimulation study. Journal of Neurophysiology, 73, 2608-2611.

Gentner, D. R., Grudin, J., \& Conway, E. (1980). Finger movements in transcription typing. DTIC document. Retrieved from http:/oai.dtic.mil.ez-proxy.brooklyn.cuny.edu: 2048/oai/oai?verb=getRecord\&metadataPrefix $=$ html\&identifier $=$ ADA085985

Gentner, D., Larochelle, S., \& Grudin, J. (1988). The roles of similarity in transfer: Separating retrievability from inferential soundness. Cognitive Psychology, 20, 524-548. 10.1016/0010-0285(88)90015-1

Grossberg, S. (1987). Competitive learning: From interactive activation to adaptive resonance. Cognitive Science, 
11, 23-63. 10.1111/j.1551-6708.1987.tb00862.x

Hurlstone, M. J., \& Hitch, G. J. (2015). How is the serial order of a spatial sequence represented? Insights from transposition latencies. Journal of Experimental Psychology: Learning, Memory, and Cognition, $41,295$.

Logan, G. D., \& Crump, M. J. C. (2011). Hierarchical control of cognitive processes. Psychology of Learning and Motivation, 54, 1-27. Retrieved from http: //linkinghub.elsevier.com.ez-proxy.brooklyn.cuny. edu:2048/retrieve/pii/B9780123855275000012

Oldfield, R. C. (1971). The assessment and analysis of hand- edness: The Edinburgh inventory. Neuropsychologia, 9, 97-113. 10.1016/0028-3932(71)90067-4

Rumelhart, D. E., \& Norman, D. A. (1982). Simulating a skilled typist: A study of skilled cognitivemotor performance. Cognitive Science, 6, 1-36. 10.1207/s15516709cog0601_1

Wilson, M. (1987). UWA Psychology: MRC Psycholinguistic Database (Dict Interface). Retrieved January 24, 2017 from http://websites.psychology. uwa.edu.au.ez-proxy.brooklyn.cuny.edu:

2048/school/MRCDatabase/uwa_mrc.htm 